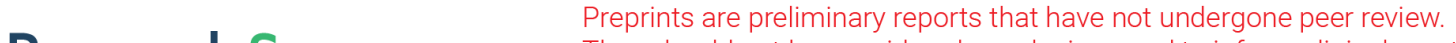 They should not be considered conclusive, used to inform clinical practice, or referenced by the media as validated information. \\ Transition from sub-Rayleigh anticrack to supershear crack propagation in snow avalanches
}

\section{Bertil Trottet}

EPFL

\section{Ron Simenhois}

Colorado Avalanche Information Center https://orcid.org/0000-0001-5572-8750

\section{Grégoire Bobillier}

WSL Institute for Snow and Avalanche Research SLF

Alec van Herwijnen

WSL Institute for Snow and Avalanche Research SLF

Chenfanfu Jiang

UCLA

Johan Gaume ( $\nabla$ johan.gaume@epfl.ch )

EPFL https://orcid.org/0000-0001-8931-752X

\section{Article}

Keywords:

Posted Date: October 22nd, 2021

DOl: https://doi.org/10.21203/rs.3.rs-963978/v1

License: (c) (i) This work is licensed under a Creative Commons Attribution 4.0 International License.

Read Full License

Version of Record: A version of this preprint was published at Nature Physics on July 25th, 2022. See the published version at https://doi.org/10.1038/s41567-022-01662-4. 


\title{
Transition from sub-Rayleigh anticrack to supershear crack propagation in snow avalanches
}

\author{
Bertil Trottet $^{1}$, Ron Simenhois ${ }^{2}$, Gregoire Bobillier ${ }^{3}$, \\ Alec van Herwijnen ${ }^{3}$, Chenfanfu Jiang ${ }^{4}$, Johan Gaume ${ }^{1,3 *}$ \\ ${ }^{1}$ École Polytechnique Fédérale de Lausanne, CH-1015 Lausanne, Switzerland \\ ${ }^{2}$ Colorado Avalanche Information Center, Boulder, CO, USA \\ ${ }^{3}$ WSL Institute for Snow and Avalanche Research SLF, \\ CH-7260 Davos, Switzerland, \\ ${ }^{4}$ University of Pennsylvania, PA-19104 Philadelphia, USA
}

*To whom correspondence should be addressed; E-mail: johan.gaume@epfl.ch

Snow slab avalanches are released following anticrack propagation in highly porous weak snow layers buried below cohesive slabs. The volumetric collapse of the weak layer leads to the closure of crack faces followed by the onset of frictional contact. Here on the basis of snow fracture experiments, full-scale avalanche measurements, and numerical simulations, we report the existence of a transition from sub-Rayleigh anticrack to supershear crack propagation involving the Burridge-Andrews mechanism. Remarkably, this transition occurs even if the shear-to-normal stress ratio is lower than the static friction coefficient as a result of the loss of frictional resistance during collapse. This finding represents a new paradigm in our understanding of snow slab avalanches presenting fundamental similarities with strike-slip earthquakes. 
After half a century of research, a consensus has been reached regarding the sequence of mechanical processes necessary for the release of snow slab avalanches $\frac{\sqrt{12} \text {. A failure is initiated }}{2}$ in a highly porous weak snow layer buried beneath a cohesive snow slab leading to mixed-mode and quasi-brittle crack propagation along the slope $\mathrm{e}^{3}$. If the slope angle is larger than the weak layer friction angle, the slab eventually slides and releases ${ }^{4}$.

For decades, a pure shear weak layer fracture was assumed ${ }^{11}$ but failed to explain field observations of crack propagation on flat terrain and remote avalanche triggering ${ }^{[6] 67}$. As a consequence, Heierli et al. ${ }^{[8}$ extended the concept of anticracks, which were originally introduced to explain deep earthquakes ${ }^{\sqrt{9}}$, to account for weak layer collapse and subsequent slab bending. Despite more recent advances reconciling these different approaches ${ }^{10}$, the slope-scale dynamics of crack propagation prior to frictional sliding still remains largely unknown. In particular, very large crack speeds $\left(>150 \mathrm{~m} \mathrm{~s}^{-1}\right)$ were evaluated based on real-scale avalanche observations by Hamre et al.11]. Although these findings only represent indirect measurements and lack validation, these large reported values allowed us to infer the potential existence of an intersonic propagation regime which contrasts with significantly lower sub-Rayleigh values measured in small-scale snow fracture experiments ${ }^{12}$. These contradictory observations suggest that there could be a transition during slope-scale fracture propagation. Transitions from sub-Rayleigh to intersonic crack velocities have been reported in earthquake dynamics based on seismological observations ${ }^{13114115}$, stick-slip ${ }^{1611718}$ and fracture experiments ${ }^{1920}$, and numerical simulations ${ }^{2122115}$. This complex process, at the frontier between fracture and friction, has been conceptualized by Burridge-Andrews ${ }^{2324}$, who showed that a daughter supershear crack can nucleate ahead of the main fracture if the local shear stress exceeds a critical value. Although snow slab avalanches have numerous mechanical features in common with earthquakes, the snow anticrack mechanism, which allows for crack propagation without an external driving shear force, further complicates analyses and prevents direct analogies. 
Here, based on numerical simulations, snow fracture experiments and full-scale avalanche measurements, we show that the onset of frictional sliding during slab avalanche release involves a transition from sub-Rayleigh anticrack to supershear crack propagation. This transition implicates the Burridge-Andrews mechanism and can occur even for slope angles smaller than the weak layer friction angle; we attribute this to the loss of frictional resistance during weak layer collapse related to the anticrack. We quantify the so-called super critical crack length associated with this supershear transition and propose a theoretical shear band model in which the collapse amplitude is accounted for by a reduced effective friction.

\section{Experimental Mismatch}

Based on elastic modulus values reported in laboratory experiments at strain rates representative of those typically involved in snow fracture tests ${ }^{25}$, the effective shear wave speed $c_{s}$ in snow is less than $120 \mathrm{~m} \mathrm{~s}^{-1}$. Crack propagation speeds obtained based on numerous small-scale $(<2$ m) snow fracture experiments vary typically between $10 \mathrm{~m} \mathrm{~s}^{-1}$ and $80 \mathrm{~m} \mathrm{~s}^{-1}$ (see Methods). However, Hamre et al. ${ }^{11}$ reported crack propagation speeds larger than $200 \mathrm{~m} \mathrm{~s}^{-1}$, based on slope-scale avalanche observations, suggesting supershear fracture. To explain these contrasting observations, the dynamics of crack propagation in weak snow layers is analyzed via numerical simulations, snow fracture experiments, and full-scale avalanche measurements.

\section{Numerical Simulations}

Numerical simulations of the so-called Propagation Saw Test (PST) ${ }^{6}$, were performed based on the Material Point Method (MPM), finite strain elastoplasticity, and a constitutive snow model accounting for weak layer volumetric collapse ${ }^{3}$ (see Methods). The PST consists of creating a crack in a predefined weak layer using a saw until it reaches a critical crack length $a_{c}$ (corresponding to a time $t_{c}$ ), after which the crack propagates in a self-sustained manner. 


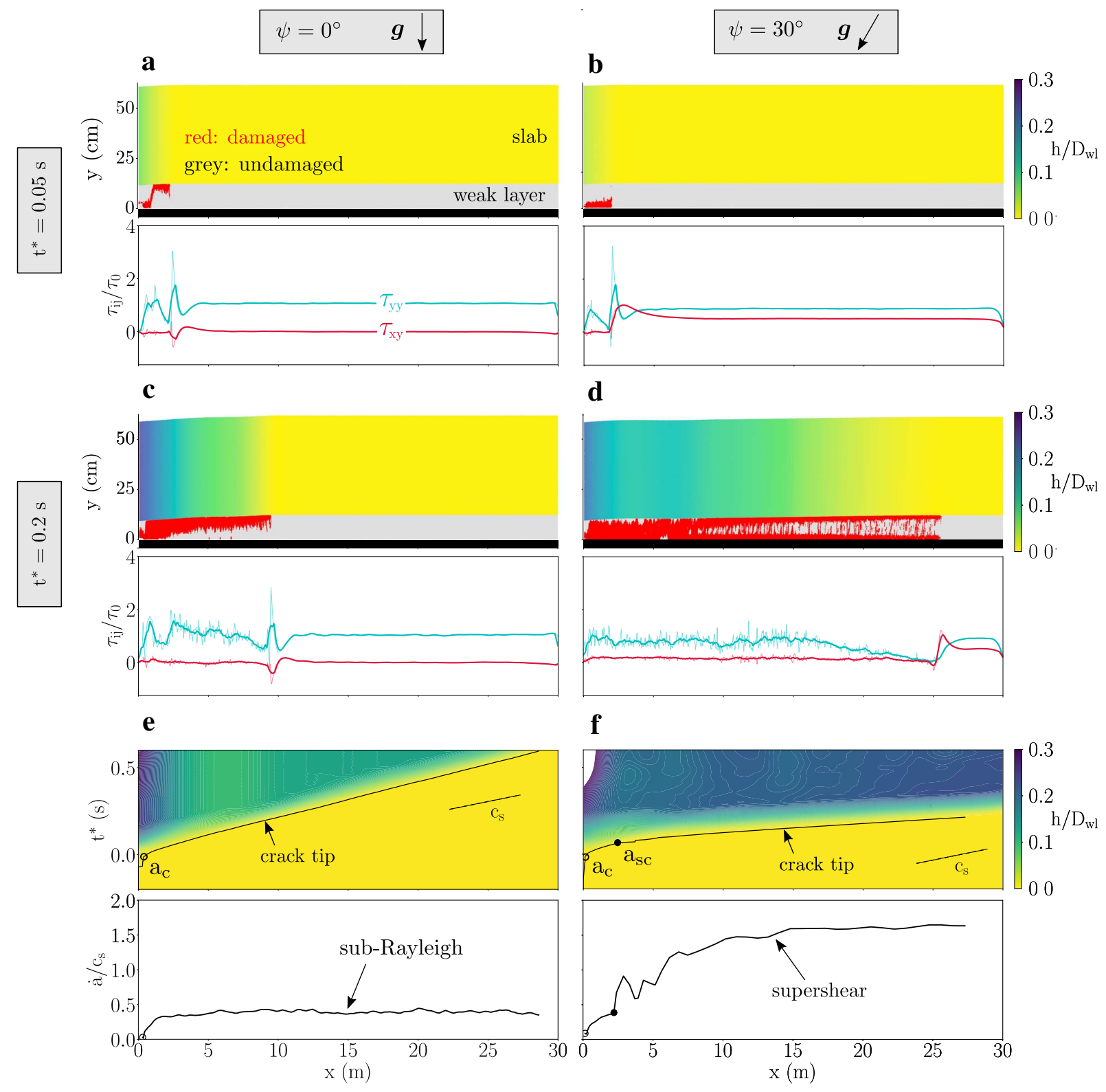

Figure 1: Crack tip morphology, dynamics, and stress state for $\psi=0^{\circ}$ (left) and $\psi=30^{\circ}$ (right). $\mathbf{a}, \mathbf{b}, t^{*}=t-t_{c}=0.05 \mathrm{~s}$ ( $t_{c}$ is the time corresponding to the onset of crack propagation). Top panels: weak layer fracture (in red) and the slab vertical displacement scaled by weak layer thickness $D_{\mathrm{wl}}$. Bottom panels: shear (red) and normal (green) stress profiles. c, d, Same as $\mathbf{a}, \mathbf{b}$, for $t^{*}=0.2 \mathrm{~s}$. e, f, Top panels: spatio-temporal evolution of $h / D_{\mathrm{wl}}$ and crack tip location. Black solid lines: crack tip position; empty circles: critical crack length $a_{c}$; filled circles: super critical crack length $a_{s c}$. Bottom panels: crack propagation speed. 
The main differences between snow fracture experiments and real avalanches are the scale and slope angle, which are generally low in experiments for practical and safety reasons. Accordingly, simulations were performed for very long PSTs, up to $140 \mathrm{~m}$, and for representative slope angles between $0^{\circ}$ and $50^{\circ}$. Figures 1 a to 1 d show weak layer fracture and the vertical displacement of the slab for flat and $30^{\circ}$ slopes, respectively (see also Supplementary Movie 1) at two different times $t^{*}=t-t_{c}$. Based on the spatio-temporal evolution of the crack tip, the crack propagation speed can be evaluated (Figs. 1e and 1f). On a flat surface, slab bending induced by weak layer collapse drives anticrack propagation. After reaching the critical crack length, the propagation speed increases until it reaches a sub-Rayleigh plateau value. Conversely, on an inclined slope, the slow increase in the crack speed is followed by a sharp transition leading to different crack propagation dynamics than observed on flat terrain. This transition occurs

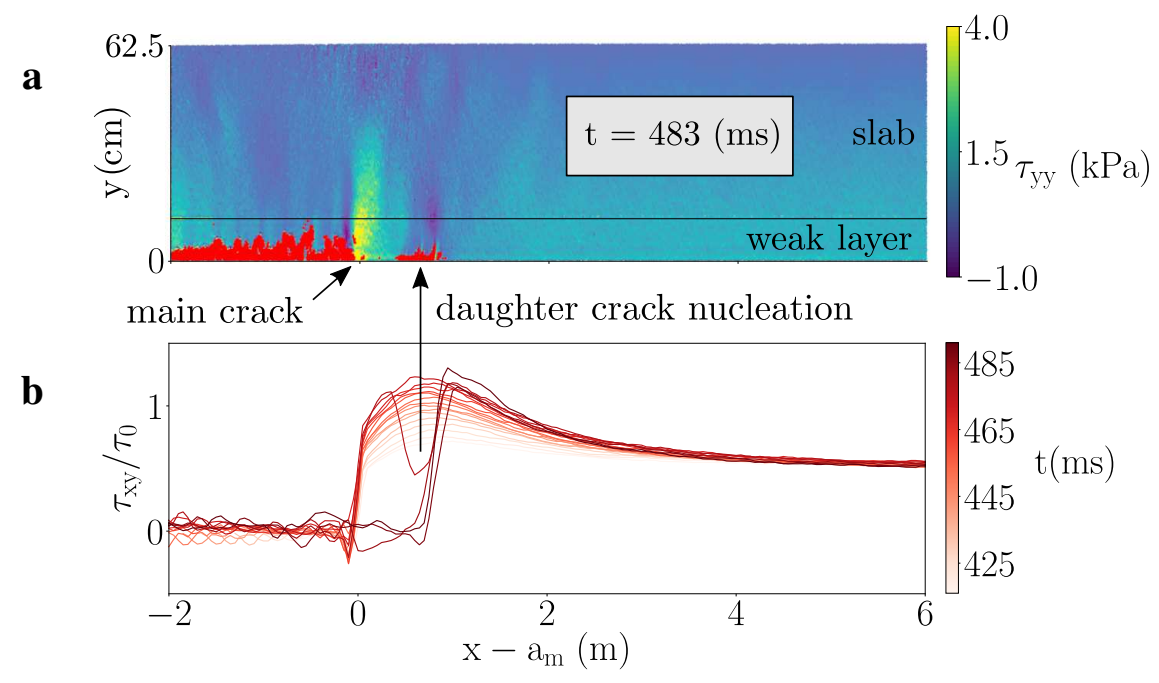

Figure 2: Illustration of the Burridge-Andrews mechanism leading to the onset of supershear crack propagation regime in the simulation shown in Fig. 1 for a $30^{\circ}$ slope. a, Visualisation of the instant of the supershear transition showing the birth of a daughter crack in front of the main crack. Cracks are shown in red and the system is additionally colored by the normal stress $\tau_{y y}$. b, Evolution of the shear stress $\tau_{x y}$ normalized by the nominal stress $\tau_{0}$ as a function of $x-a_{m}$ ( $x$ : longitudinal position; $t$ : time; $a_{m}$ : position of the main crack). 
for a "super critical crack length" $a_{s c}$, which is significantly larger than $a_{c}$, and starts with the nucleation of a daughter crack in front of the main fracture (Fig. 2a). This topological change results in an apparent jerk in the crack length followed by a sharp increase in the crack speed to an intersonic regime that then converges to a speed of $1.6 c_{s} \approx(1+2 \nu)^{1 / 2} c_{s}=\sqrt{E / \rho}$, where $E, \nu$ and $\rho$ are the slab elastic modulus, Poisson's ratio and density, respectively. Note that, in the analysis of our simulations and the evaluation of the crack speed, the hypothesis of crack uniqueness is made. Accordingly, the crack tip is defined as the location of the furthest plasticized particle. Based on our single crack tip definition, the Burridge-Andrews mechanism necessarily implies an infinite propagation speed after reaching $a_{s c}$, which explains the reported sudden jerk. In principle and as described by Liu and Lapusta ${ }^{26}$, the daughter crack (Fig. 2) spontaneously propagates as a supershear crack in both directions and then merges with the main crack. Once supershear propagation occurs, the crack spontaneously branches leading to complex micro-cracks patterns as reported by Sharon et al. 27 .

\section{Onset of Supershear Fracture}

Crucial insights with respect to the mechanical origin of this transition are found by examining the stress state in the weak layer (Figs. $1 \mathrm{a}$ to $1 \mathrm{~d}$ ). On flat terrain, bending of the slab induces stress concentrations at the crack tip and a negative shear stress (directed up-slope). At the crack tip, the normal stress, higher than the shear stress, drives anticrack propagation. The stress profile in the vicinity of the crack tip remains approximately self-similar throughout the entire simulation (Figs. 19a and 17). Behind the crack, the normal stress fluctuates around the far field value, corresponding to the new contacts between the crack faces. On the slope, slab tension induced by the slope-parallel component of the gravitational force overcomes the stresses induced by slab bending, leading to a positive shear stress peak at the crack tip (directed down-slope). Shear and normal stress are initially of the same order of magnitude, and both 
drive mixed-mode anticrack propagation. After reaching the super critical crack length, a clear and sharp transition of the stress profile in the vicinity of the crack tip is observed (see Figs. 1 1 , $1 \mathrm{~d}$ and $2 \mathrm{p}$ ). The normal stress becomes very low, and the crack is driven by shear. Therefore, the transition from sub-Rayleigh to intersonic crack speeds is always accompanied by a transition from mixed-mode anticrack to pure mode II crack propagation. Under these conditions, the super critical crack length can be described by a modified shear band propagation model under the effect of strain softening $28[29$ and weak layer collapse (see Methods):

$$
a_{s c}=\Lambda \frac{\tau_{p}-\tau_{g}}{\tau_{g}-\tau_{r}^{*}}
$$

where $\Lambda$ is a characteristic elastic length related to the elasticity and height of the two layers, $\tau_{p}$ is the weak layer shear strength, $\tau_{g}=\tau_{0} \sin \psi$ is the shear stress induced by the slab nominal load $\tau_{0}$, and $\tau_{r}^{*}=\tau_{0} \cos \psi \tan \phi^{*}$ is the shear band effective residual stress and is a function of both the slab load and the effective friction angle $\phi^{*}$, which depends on the weak layer friction angle and the collapse height (Fig. 3). It should be noted that our super critical crack length $a_{s c}$ presents similarities with the length proposed in previous studies on slip instabilities (same

trend with slope angle and divergence close to friction angle as in Andrews ${ }^{24}$ and Kammer et al. $\left.{ }^{21}\right)$. Yet, the bi-layer configuration studied here prevents a direct analogy between these different formulations.

\section{Collapse-Dependent Friction Weakening}

Numerous numerical simulations were performed for different slope angles and mechanical properties of both slab and weak layer (see Methods), to evaluate the condition for the onset of anticrack $\left(a_{c}\right)$ and supershear $\left(a_{s c}\right)$ propagation. The critical crack length decreases with increasing slope angle $\psi$ and is of the order of $0.1 \Lambda$ (Fig. 3). The super critical crack length only exists if the slope angle $\psi$ is larger than the effective friction angle $\phi^{*}$. It also decreases with increasing slope angle and varies between $0.5 \Lambda$ and $15 \Lambda$ in the simulations. The effective 


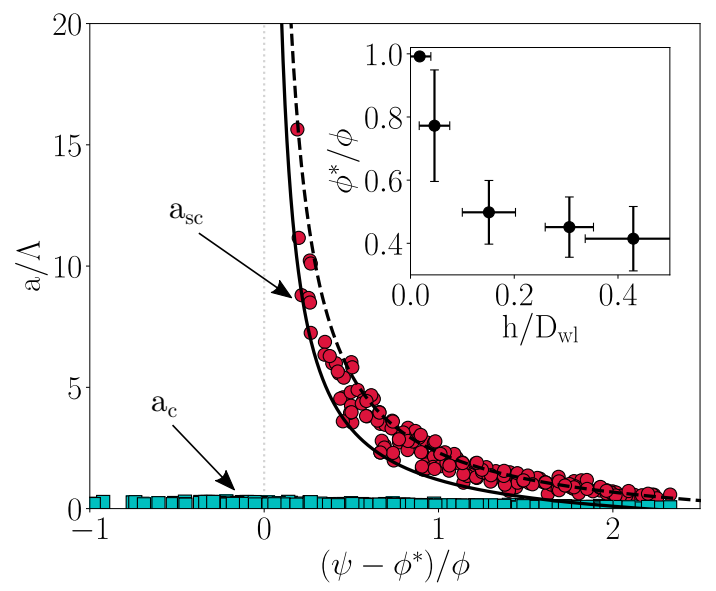

Figure 3: Simulation of critical (green squares) and super critical (red circles) crack lengths normalized by the elastic length $\Lambda$ as a function of the normalized slope angle $\left(\psi-\phi^{*}\right) / \phi$. The solid and dashed lines correspond to theoretical curves for apparent friction angles of $\phi^{*}=\phi$ and $\phi^{*}=0.4$, respectively. The inset shows $\phi^{*} / \phi$ as a function of the collapse amplitude $\mathrm{h}$ normalized by weak layer thickness $\mathrm{D}_{\mathrm{wl}}$.

friction coefficient controls the onset of the supershear transition and significantly depends on the collapse amplitude $h$ of the weak layer (inset of Fig. 3). Without volumetric collapse, the effective friction angle is exactly equal to the friction angle. However, increasing collapse heights reduce the apparent frictional resistance of the shear band as reported by van Herwijnen and Heierli ${ }^{4}$. This local friction reduction enables a supershear transition for slope angles lower than the weak layer friction angle. In effect, once the crack reaches its super critical crack length, its sharp acceleration is associated with a significant increase of the slab section non supported by the weak layer, leading to unstable propagation even below the friction angle. The simulation data are well reproduced by Eq. (1) for an effective friction angle between $0.4 \phi$ and $\phi$. 

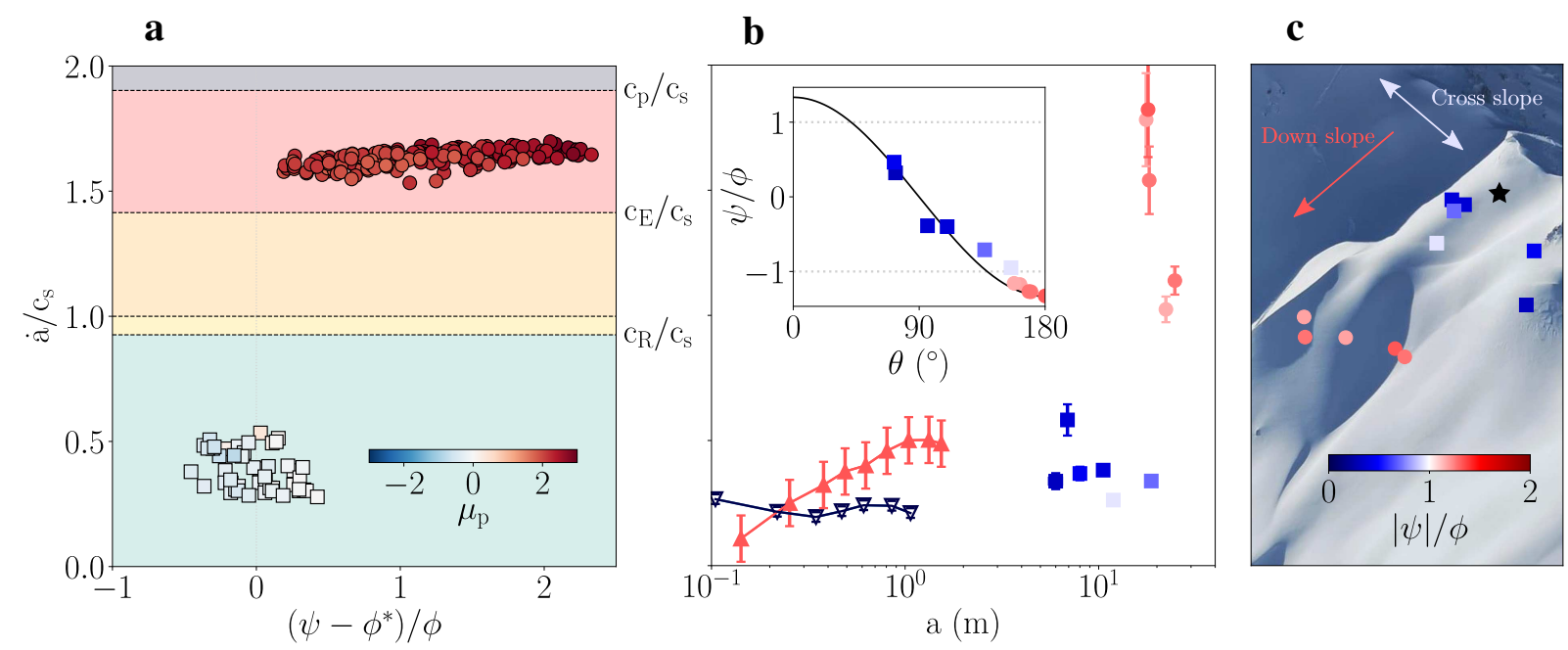

Figure 4: a, Asymptotic crack speeds obtained in numerical simulations. Points are colored according to the peak shear-to-normal stress ratio $\mu_{p}$. The background color represents different propagation regimes limited by the Rayleigh wave speed $c_{R}$, the shear wave speed $c_{s}$, the Eshelby wave speed $c_{E}=\sqrt{2} c_{s}$ and the p-wave speed $c_{p}$. b. Crack speeds obtained in field experiments (PST, triangles) and full-scale avalanche measurements (squares and circles). The inset represents the normalized slope angle versus the azimuthal angle $\theta$, characterizing crack direction (down-slope or cross-slope). c, Location of the measurement points on the slope prior to the avalanche.

\section{Propagation Speed Regimes and Validation}

The asymptotic crack propagation speeds obtained in all simulations are shown in Fig. 4a. For slope angles lower than the effective friction coefficient, the asymptotic propagation speed is sub-Rayleigh and varies between $0.25 c_{s}$ and $0.6 c_{s}$. In this regime, the peak shear-to normal stress ratio $\mu_{p}$ is low due to large normal stresses at the peak (Fig. 11 c) and change in sign according to the shear stress nature (i.e., negative when slab bending is dominant and positive when slab tension is dominant). For larger slope angles, the propagation speed switches to intersonic and approaches a value of $1.6 c_{s} \sim \sqrt{E / \rho}$, similar to the longitudinal elastic wave speed in a beam. In this case, the large values of $\mu_{p}$ indicate that this regime is driven by large shear stress and low normal stress values (Figs. 1 $1 \mathrm{~d}$ and 2 ) resulting from slab tension. 
Crack propagation speeds obtained in two representative snow fracture experiments (see Methods) performed on flat terrain and on a $30^{\circ}$ slope indicate sub-Rayleigh propagation with speeds (Fig. 4b) in line with those obtained in the numerical simulations in this regime. The slightly higher sub-Rayleigh speed obtained in the high-angle experiment highlights that this transition requires both a steep slope and a crack length that reaches $a_{s c}$. In this tilted experiment, based on Eq. (1) and the reported relative collapse amplitude of $6 \%, a_{s c}$ is larger than the beam length typically used in this test $(\sim 1.5 \mathrm{~m})$. As a consequence, classical snow fracture experiments (i.e. propagation saw tests ${ }^{30 \mid 31}$ ) are not well suited to characterize this transition, which requires slope-scale dimensions. An analysis of a full-scale deep slab avalanche triggered in 2019 in Wallis, Switzerland, on a $42^{\circ}$ slope allows us to experimentally confirm the existence of an intersonic propagation regime and to further characterize crucial spatial characteristics. Based on an analysis of the change in frame pixel intensity (see Methods and Supplementary Movies 2 and 3), the crack propagation speeds can be measured at different key points (Figs. $4 \mathrm{~b}$ and $4 \mathrm{c}$ ) in directions varying between down-slope and cross-slope (characterized by the azimuthal angle $\theta$ ). Crack speeds in the down-slope direction (mode II) are intersonic with values in good agreement with the asymptotic values obtained in steep and long numerical PSTs. Conversely, cross-slope (mode III) and sub-Rayleigh speeds are in good agreement with the asymptotic values obtained in low-angle numerical simulations, as well as experimental PSTs.

\section{Discussion}

The findings we report have important consequences for the size of the release zone and therefore hazard evaluation. By accounting for slab tensile failure, we show (see Extended Data Fig. 8) that soft slabs with low tensile strengths prevent the transition from occurring. This leads to slab fracture that starts from the snowpack surface, where the tensile stress is the highest, and occasionally to the so-called "en-echelon" characteristic. In this case, the propagation 
is substantially affected by slab fracture, leading to crack arrest (see Fig. S3) and therefore to small release areas. For denser slabs with higher tensile strength, slab fractures do no significantly affect crack propagation, and the occurrence of the supershear transition. Release areas can therefore be much larger, and likely mostly constrained by topography (e.g. changes in slope angle). Hence, the local stress perturbation caused by the crack progression induces the slab fracture to starts from the weak layer interface. These implications of our results are consistent with observations of (i) slab fractures initiated at the surface in small-scale fracture experiments $^{\sqrt{32}}$ and indirect observations of slab fracture initiated at the bottom of snowpacks in real avalanches ${ }^{33}$ and (ii) soft slab avalanches typically being smaller than hard ones ${ }^{3477}$.

Hazard management procedures in mountainous regions still rely on empirical evaluations of the avalanche release size. The new paradigm reported here suggests that a pure shear model is sufficient to predict the sizes of large slab avalanches. Therefore, considering depth-averaged pure shear release models would allow the present work to be brought to an operational level, improving avalanche risk assessment and forecasting.

Furthermore, our findings indicate that the crack propagation speed measured in small-scale experiments is not necessarily representative of crack speeds on real avalanche terrain in the down/up-slope direction (mode II). In fact, despite the different propagation mechanisms, the experimentally measured values are in good agreement with the avalanche cross-slope propagation speeds (mode III, Fig. 4b) which is theoretically limited by the Rayleigh wave speed ${ }^{23}$. Here, we provide a two-dimensional theoretical framework that allows the conditions for the onset of this transition to be evaluated, as well as the crack propagation speed, which depends on slab elastic waves. Future work should include slope-scale experiments and simulations to study three-dimensional propagation patterns ${ }^{35}$, as well as the complex interplay between the weak layer and slab fracture during the release process.

Our findings shed light on a previously unreported stage of the avalanche release process 
with key implications for predictions of avalanche danger. More generally, our results reinforce the analogy between snow slab avalanches and earthquakes. While the mechanism of supershear propagation has been rarely reported in large strike-slip earthquakes ${ }^{15}$, it requires a very common combination of topographical and mechanical ingredients in slab avalanche release.

\section{Materials and Methods}

\section{Snow fracture experiments}

Two hundred twenty two snow fracture experiments (so-called Propagation Saw Tests or PSTs) were conducted in Davos, Switzerland during Winter 2015-2016 (Fig. 5). These experiments

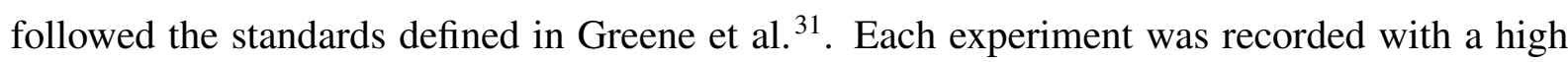
speed camera allowing to compute, the displacement of black markers inserted in the pit wall using Particle Tracking Velocimetry (PTV) ${ }^{[12}$ with an accuracy of about $0.1 \mathrm{~mm}$. Crack propagation velocity was measured based on the temporal delay between the onset of vertical motion of the markers based on the average speed computed from 6 vertical displacement threshold of $0.07,0.08,0.09,1,1.1$, and $1.2 \mathrm{~mm}$. Two PSTs from this large dataset were selected for in-depth analysis. Both experiments have similar mechanical and geometrical properties presented in Table 1. The standard deviations of PSTs presented in Fig. 4 were computed from the mean speed deviation based on all thresholds. The main difference is the slope angle as one experiment was performed on flat terrain while the other was conducted on a $37^{\circ}$ slope. Crack propagation speeds obtained in these two experiments $\left(\dot{a} / c_{s} \sim 0.2\right.$ in PST 1 and $\dot{a} / c_{s} \sim 0.4$ in PST 2) are representative of that obtained in the 222 PSTs, as shown in Fig. 5.

\section{Full scale avalanche measurements}

On January 31, 2019, a professional snowboarder triggered a dry-snow slab avalanche (Fig. 11a) in a location nearby of "Col du Cou" in Wallis, Switzerland (Fig. 2a). A few minutes before, the group checked the snowpack stability on a slope right behind and did not trigger an avalanche 

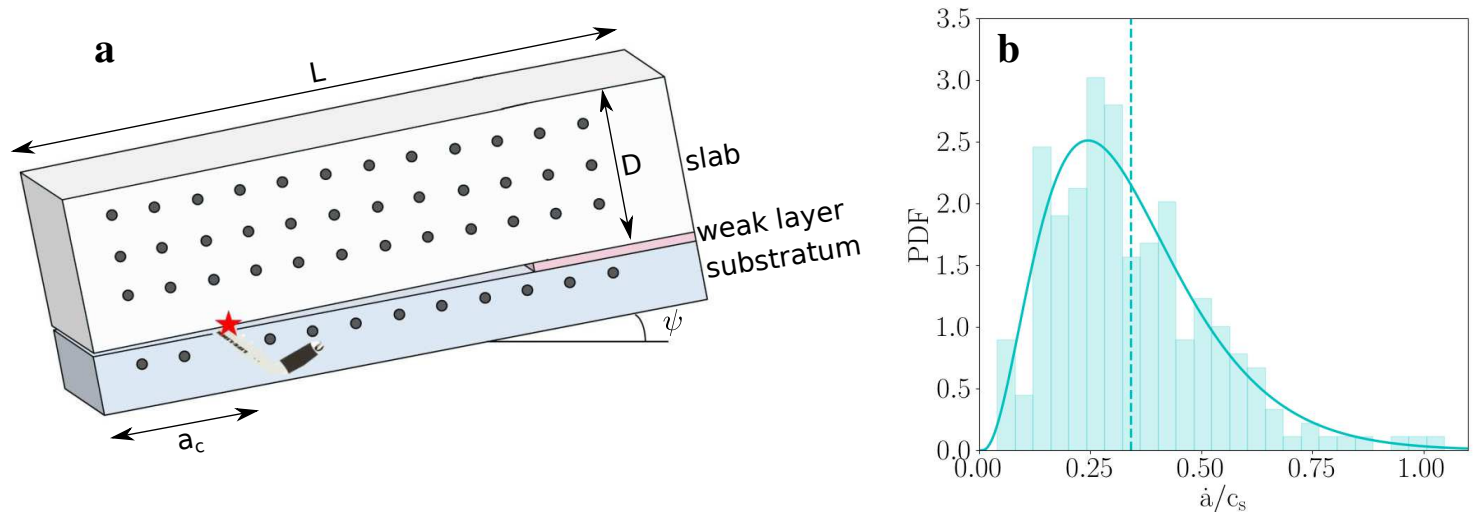

Figure 5: a, Schematic of the Propagation Saw Test. b, Histogram of the normalized crack propagation speeds obtained from 222 PSTs following a beta law probability density distribution. The dashed line corresponds to the median of the distribution.

\begin{tabular}{lrr} 
& PST 1 & PST 2 \\
\hline Slab thickness $D(\mathrm{~cm})$ & 70 & 75 \\
Weak layer thickness $D_{\mathrm{wl}}(\mathrm{cm})$ & 7.5 & 15 \\
Length $L(\mathrm{~cm})$ & 110 & 160 \\
Slope angle $\psi\left({ }^{\circ}\right)$ & 0 & 37 \\
Mean slab density $\rho^{\text {init. }}\left(\mathrm{kg} \cdot \mathrm{m}^{-3}\right)$ & 279 & 255 \\
\hline
\end{tabular}

Table 1: Geometrical and mechanical properties of the Propagation Saw Test experiments.

(see ski tracks in Fig. 11B). The snowboarder triggered this slab avalanche, very likely because of the large impact force induced by a jump from the ridge (see Supplementary Movie 3), which in turn led to failure initiation and crack propagation in the buried weak snow layer close to the ground.

The avalanche was recorded using a high-speed video camera (frame rate: 120 frames per second) allowing analysis of slab motion induced by crack propagation within the buried weak snow layer. Our crack propagation speed measurements rely on four stages: i) video stabilization using Optical Flow, ii) Eulerian Magnification to enhance small changes in snow reflection due to slab deformation, and detection of iii) time and iv) location of slab deformation between video frames. After determining the location and timing of slab deformation, we calculated the deformation distances and time difference from the crack initiation point (impact of the snow- 
boarder) and time and accounted spatial and temporal uncertainties. This allow us to drive an estimation of the average crack propagation speed in the determined direction.

We used Lucas and Kanade Optical flow algorithms ${ }^{\sqrt{36}}$ to stabilize camera movements during the video clip to generate motion vectors of pixels around static terrain features like rocks and snow roughness around the avalanche release area between video frames. We used the inverse optical flow motion to move the video frames and stabilize the scene where every point on the slope remains at the same pixel location throughout the video clip.

Eulerian Video Magnification is a method to enhance small color changes in videos that otherwise are invisible to the naked eye $\mathrm{e}^{37}$. This method has two main steps; it applies spatial decomposition on the video frames and applies a temporal filter to the frame sequence. Subsequently, the filtered band for the targeted frequency range is amplified by a predetermined factor. Finally, the amplified down-sampled bands are added to the original signal, and then the pyramid is collapsed to create the magnified signal. We compared the frequency bands in areas where the avalanche was released to areas where it was not released to filter out frequencies associated with noise. We used frequencies between 1.5 and $4.3 \mathrm{~Hz}$, and the amplifying color magnification factor of 50 .

We used the difference in pixel intensity between two consecutive frames to detect slab deformation's start time and location during the avalanche release. To filter out noise, we considered only areas where the pixel intensity changes between frames exceeded the changes in areas where the avalanche did not release. The coordinates of the video's points of interest were obtained using a Digital Elevation Model with a $0.25 \mathrm{~m}$ resolution and matching key points of the slope (ridge and rocks, Fig. 11). We estimate the error of crack initiation time and the onset of slab deformations within one frame with a mean error of $0.02 \mathrm{sec}$ and a standard deviation of $0.01 \mathrm{sec}$. We estimate distance measurements error due to the spatial decomposition in the Eulerian magnification processing and the conversion from video frames to lat/long coordinates 
to be on average of $0.46 \mathrm{~m}$ with a standard deviation of $0.07 \mathrm{~m}$ (ridge and rocks, Fig. 1).

The density of the slab was estimated separately using two techniques: i) based on the hand hardness and grain size obtained from a manual snow profile performed one day after the avalanche at Hohsaas station in Saas Grund (VS) distant of $10 \mathrm{~km}$ from the avalanche event; ii) based on a snow cover simulation (SNOWPACK ${ }^{38}$ ) and meteorological data available at the Orzival IMIS meteorological station, around $3 \mathrm{~km}$ from the avalanche. The manual snow profile is given in Fig. 2. Based on Geldetzer and Jamieson ${ }^{39}$, an average slab density of $250 \mathrm{~kg} \mathrm{~m}^{-3}$ $\left(+/-40 \mathrm{~kg} \mathrm{~m}^{-3}\right)$ was evaluated. The SNOWPACK simulation gives an average slab density for the lowest depth hoar weak layers of about $250 \mathrm{~kg} \mathrm{~m}^{-3}$, in good agreement with the estimation based on the manual snow profile. For the sake of computing the slab elastic modulus and elastic wave speeds, a slab density of $250 \mathrm{~kg} \mathrm{~m}^{-3}$ was thus chosen.

\section{Elastic wave speeds in snow}

The mechanical properties of snow are time dependent, and with increasing strain rates the strength decreases while the elastic modulus increases (e.g. Schweizer and Camponovo ${ }^{40}$ ). In addition, the ice matrix in snow has a highly disordered, cohesive-granular microstructure. As such, snow microstructure plays a crucial role in the rich, rate- and temperature-dependent behavior observed in snow (e.g. Narita ${ }^{41}$; Schweizer and Camponovo ${ }^{40}$ ). It is known that density alone is not sufficient to describe snow mechanical properties, as for a given density, values scatter by orders of magnitude due to differences in snow microstructure (e.g. Mellor ${ }^{\sqrt{42}}$ ). Furthermore, in slab avalanche release, the snow slab is usually composed of multiple layers with different snow types. To model the complete physics of snow slab avalanche release would thus require accounting for temperature, strain rate, snow density and microstructure. As many of the processes in snow mechanics are still poorly understood, the slab is therefore usually modelled as a homogeneous layer with a bulk density and an effective elastic modulus ${ }^{30}$. Therefore, to 
compute the speed of the elastic waves in snow, we used an approximation for the effective elastic modulus of the slab based on density according to the laboratory experiments of Sigrist ${ }^{25}$, performed at high strain rates which are typically involved in snow fracture experiments. The following power-law relationship was used:

$$
E=9.68 \cdot 10^{8}\left(\frac{\rho}{\rho_{\text {ice }}}\right)^{2.94}
$$

with $\rho_{\text {ice }}=917 \mathrm{~kg} \cdot \mathrm{m}^{-3}$. In addition, we used a representative Poisson's ratio ${ }^{43}$ of $\nu=0.3$.

\section{Numerical model and simulation setup}

The Material Point Method is a hybrid Lagrangian-Eulerian numerical technique, where the Lagrangian particles track history dependent variables such as position, velocity, and deformation gradient, while the Eulerian grid enables the computation of spatial gradients of these quantities. The transfer of information between the grid and the particles is then handled by an interpolation scheme. The time is discretized using a symplectic Euler time integrator. The

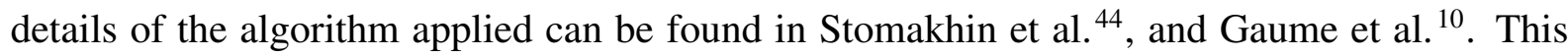
algorithm is particularly suitable to handle large topological changes and allows to resolve the governing equations of continuum mechanics 3, 4, where $\rho$ is density, $t$ is time, $\mathbf{v}$ is velocity, $\sigma$ is the Cauchy stress tensor and $\mathrm{g}$ is the gravitational acceleration.

$$
\begin{gathered}
\frac{D \rho}{D t}+\rho \nabla \cdot \mathbf{v}=0 \\
\rho \frac{D \mathbf{v}}{D t}=\nabla \cdot \boldsymbol{\sigma}+\rho \mathbf{g}
\end{gathered}
$$

In the scope of finite strain elastoplasticity, the Cauchy stress $\sigma$ can be related to the strain through an elastoplastic constitutive law, where $\mathbf{F}^{E}$ denotes the elastic part of the deformation gradient $\mathbf{F}$.

$$
\boldsymbol{\sigma}=\frac{1}{\operatorname{det} \mathbf{F}} \frac{\partial \Psi}{\partial \mathbf{F}^{E}} \mathbf{F}^{E}
$$


where $\Psi$ is the elastoplastic potential energy density related to the Lamé coefficients based on the St-Venant-Kirchhoff hyperelastic model. A similar constitutive model to the one proposed by ${ }^{10}$ is used in this study to simulate the behavior of both the slab and the weak layer. It is based on an associative Cohesive Cam Clay (CCC) yield surface and a softening-hardening law which depends of the material type (i.e. weak layer, slab). The yield surface is expressed as

$$
y(p, q)=q^{2}(1+2 \beta)+M^{2}\left(p+\beta p_{0}\right)\left(p-p_{0}\right)
$$

where $p$ is the pressure defined as $p=\operatorname{tr}(\tau) / d, q$ is the von Mises equivalent stress defined as $q=\sqrt{3 / 2 s: s}$, and $s=\boldsymbol{\tau}+p \boldsymbol{I}$ is the deviatoric stress tensor and $\boldsymbol{I}$ is the identity matrix, $p_{0}$ is the compressive strength, $\beta p_{0}$ the tensile strength, and $M$ is the slope of the material critical state line without cohesion. For the slab, $p_{0}=p_{0}^{\text {slab }}$ may increase (hardening) of decrease (softening) depending on the amount of plastic deformation according to Eq. 7 ;

$$
p_{0}^{\text {slab }}=k_{1} \sinh \left(\max \left(-\epsilon_{v}^{P}, 0\right)\right)
$$

with $k_{1}$, the bulk modulus associated to a Young's modulus of $1 \mathrm{MPa}$ (kept constant in this study), and $\epsilon_{v}^{P}$ is the volumetric plastic deformation. For the weak layer, $p_{0}=p_{0}^{\mathrm{wl}}$ is defined according to

$$
p_{0}^{\mathrm{wl}}=k_{1} \sinh (\max (-\eta, 0))
$$

where $\eta$ is the anticrack plastic strain defined in Gaume et al $\frac{10}{10}$ according to

$$
\dot{\eta}= \begin{cases}\alpha\left|\dot{\epsilon}_{v}^{P}\right|, & \text { if } t \leq t_{c} \\ \xi \dot{\epsilon}_{v}^{P}, & \text { if } t>t_{c}\end{cases}
$$

for the weak layer. In the above equations, $t_{c}$ is the time corresponding to complete softening i.e. $p_{0}=0$, time at which $\beta$ is set to zero making the behavior of the weak layer cohesionless. $\alpha$ is a softening coefficient, $\xi$ is a hardening coefficient, . The main difference with the weak layer model developed by Gaume et al. ${ }^{10}$ lies in the fact that the softening and the hardening behavior 
are now independent of each other. In addition, the constant pre-factor $k_{1}$ allows to control the amount of volumetric collapse based on $\xi$ only, independently of the elastic parameters. Hence, the collapse height of the weak layer can be analytically predicted based on Eqs. (10) and (9) as a unique function of $\xi$ as follows:

$$
\frac{h}{D_{\mathrm{wl}}}=1-\exp \left(\epsilon_{v}^{P}\right)=1-\exp \left(\frac{1}{\xi} \sinh ^{-1}\left(\frac{p_{0}}{k_{1}}\right)\right)
$$

Simulation results for different values of $\xi$ are shown in Fig. S8 together with its analytical counterpart.

The numerical setup consists of a Propagation Saw Test with a bilayer system composed of a cohesive elastic slab and an elastoplastic weak layer of respectively 50 and $12.5 \mathrm{~cm}$ thick. A crack of length $a$ is created in the weak layer by a virtual saw (which sets the elastic modulus of particles in its neighborhood to zero) until it propagates spontaneously once a critical crack length $a_{c}$ is reached. The saw advances at a velocity of $1 \mathrm{~m} \mathrm{~s}^{-1}$, speed which was verified to be sufficiently low not to affect the presented results. We used the material point method to perform 2D simulations of PSTs of length ranging from 25 to $140 \mathrm{~m}$. This length was varied to ensure a steady crack propagation regime amongst all our simulations. The constitutive models previously described were used for the slab and the weak layer. The position of the particles at the bottom of the weak layer are fixed. Table 2 shows a synthesis of the parameter values used in this study. To evaluate the shear strength of the material for given nominal load state (see below), we performed additional simulations for loading angles from 0 to $180^{\circ}$. This allowed to obtain the weak layer shear strength as a function of the normal stress. Based on the Critical State Line concept, the friction angle $\phi$ of the damaged weak layer can be directly related to the yield surface parameters following Eq. 11 and equal to $21^{\circ}$ which is lightly lower in the exposed simulations than the one reported by Herwijnen et al. ${ }^{4}$.

$$
\phi=\arcsin \frac{3 M}{6+M}
$$




\begin{tabular}{llrr} 
& & Slab & Weak Layer \\
\hline Geometry & Thickness $D(\mathrm{~cm})$ & 50 & 12.5 \\
& Length $^{1} L(\mathrm{~m})$ & $25-140$ & $25-140$ \\
& Slope angle $^{2} \psi\left(^{\circ}\right)$ & $0-50$ & $0-50$ \\
\hline Material & Initial density $\rho^{\text {init. }}\left(\mathrm{kg} \cdot \mathrm{m}^{-3}\right)$ & 250 & 100 \\
& Young's modulus $^{3} E(\mathrm{MPa})$ & $5-30$ & $0.5-2$ \\
& Poisson's ratio $\nu$ & 0.3 & 0.3 \\
& Initial consolidation pressure $p_{0}^{\text {init. }}(\mathrm{kPa})$ & $13.9-47.5$ & 13 \\
& Tension/compression ratio $\beta$ & 0.8 & 0.2 \\
& Friction coefficient $M$ & 0.8 & 0.8 \\
& Hardening factor & \\
& Softening factor $\alpha$ & 10 & $0.0025-0.1$ \\
& Element size $d x(\mathrm{~cm})$ & - & 500 \\
\hline CFL & \multicolumn{2}{c}{1} & \\
& Frame rate FPS $\left(\mathrm{s}^{-1}\right)$ & 0.5 & \\
\hline
\end{tabular}

${ }^{1} L$ values includes 25, 30, 40, 50, 60, 80, 100, 120 and 140.

${ }^{2} \psi$ values includes $0,5,10,15,20,25,30,35,40,45$ and 50 .

${ }^{3} E_{\text {slab }}$ values includes 5, 10, 15, 20, 25, and 30. $E_{\mathrm{wl}}$ values includes $0.5,1$ and 2.

${ }^{4} \xi$ values includes $0.0025,0.005,0.01,0.05$ and 0.1 .

Table 2: Parameters values employed in the MPM simulations.

Quantities of interest such as the positions $(\mathbf{x})$, the Kirchoff stress tensor $(\boldsymbol{\tau})$, and the volumetric plastic strain $\left(\epsilon_{v}^{P}\right)$ of each particles are outputted. We can then track the crack length $(a)$ defined as the $x$-position of the furthest plasticised particle. In parallel, the longitudinal evolution of the collapse height $(h)$ and of the Kirchhoff stress tensor components $\left(\tau_{i j}\right)$ of both slab and weak layer are computed by averaging them on slices of $d l=5 d x$ width. The crack tip if defined as the location of the furthest plasticized particle (i.e. the furthest particle presenting a plastic deformation). The computation of the crack propagation speed $(\dot{a})$ is directly obtained from the longitudinal evolution of the crack tip position. The critical crack length $\left(a_{c}\right)$ is computed as the position of the saw for which the crack starts to self-propagate over a length larger than $10 \mathrm{~cm}$ ahead of the saw. The supercritical crack length $\left(a_{s c}\right)$ is evaluated from the crack propagation speed profile as a three step approach. First, we search the location of the maximum local 
variation of the crack speed over a moving average of 4 frames according to a rolling median algorithm, this allow us to detect the acceleration of the propagation following the first peak. Based on this detection, the domain is resized and the algorithm is applied a second time to detect the ascending side of the peak which allows a second resize of the domain. This enables us to evaluate a suitable temporal window for the search of the super critical crack length $a_{s c}$. Third, $a_{s c}$ is estimated as the position corresponding to the maximum jerk (time derivative of the acceleration) within this window. The asymptotic crack propagation speed is computed from the average speed at the end of the propagation over a distance of $5 \Lambda$, ensuring that the propagation reaches a permanent regime.

\section{Analytical model for the onset of supershear fracture}

After the supershear transition, the crack propagates in mode II (Fig. 1). Hence, we describe the onset of supershear transition based on a shear band propagation model to predict the super critical crack length. The approach used is similar to that of McClung ${ }^{45}$, Chiaia et al. ${ }^{46}$ and Gaume et al ${ }^{29}$ but introduces the effect of the collapse height on the residual shear friction.

As first approximation, we neglect inertia. We neglect the size of the process zone and the effect of slab bending. We assume continuity of displacements at slab-weak layer interface and homogeneous shear of the weak layer in $y$-direction. In addition, once failed, the weak layer has a constant residual shear strength $\tau_{r}^{*}$ which depends on the collapse height. For a crack of length $a$ with a tip located in $x=0$ (Extended Data Figure 5), it is possible to relate the stress field $\tau(x)$ to the displacement field $u(x)$ as

$$
\tau(x)= \begin{cases}\tau_{r}^{*} & \forall x<0 \\ -G_{\mathrm{wl}} \frac{u(x)}{D_{\mathrm{wl}}} & \forall x \geq 0\end{cases}
$$

where $G_{\mathrm{wl}}$ and $D_{\mathrm{wl}}$ are the weak layer shear modulus and thickness, respectively. Considering the force balance in the $x$-direction of an elementary volume of length $d x$, it is possible to derive 
the local force balance acting on this local slab section as

$$
\frac{d N}{d x}+\tau(x)-\tau_{g}=0
$$

in which $\tau_{g}=\rho g D \sin \psi$ and $N$ is the slab tensile force given by the plain strain elastic relationship $N(x)=E^{\prime} D \frac{d u}{d x}$, with $E^{\prime}=E /\left(1-\nu^{2}\right)$ leading to the following second order equation

$$
\frac{d^{2} u}{d x^{2}}-\frac{u(x)}{\Lambda^{2}}-\frac{\tau_{g}}{E^{\prime} D}=0 \forall x \geq 0
$$

with $\Lambda=\sqrt{\frac{E^{\prime} D D_{\mathrm{wl}}}{G_{\mathrm{wl}}}}$ and which has the well-know general solution

$$
u(x)=c_{1} e^{x / \Lambda}+c_{2} e^{-x / \Lambda}-\frac{\tau_{g}}{E^{\prime} D} \Lambda^{2}
$$

The two constants $c_{1}$ and $c_{2}$ can be evaluated from boundary conditions. First, tension in the slab far from the crack vanishes which leads to $c_{1}=0$. Second, we assume a constant and homogeneous effective residual stress which allows us to compute slab tension at the crack tip, i.e., $N(x=0)=\left(\tau_{g}-\tau_{r}^{*}\right) a$ leading to $c_{2}=-\Lambda \frac{\left(\tau_{g}-\tau_{r}^{*}\right) a}{E^{\prime} D}$. As a consequence, one can obtain the profile of the weak layer shear stress as follows:

$$
\tau(x)= \begin{cases}\tau_{g}\left(1+\frac{a}{\Lambda}\left(1-\frac{\tau_{r}^{*}}{\tau_{g}}\right) e^{-\frac{x}{\Lambda}}\right) & \forall x \geq 0 \\ \tau_{r}^{*} & \forall x<0\end{cases}
$$

One can thus determine the super critical crack length $a_{s c}$ based on the shear stress at the crack tip $(x=0)$ and the shear strength of the weak layer in mode II $\tau_{p}$ as:

$$
a_{s c}=\Lambda \frac{\tau_{p}-\tau_{g}}{\tau_{g}-\tau_{r}^{*}}, \quad \tau_{g}=\rho g D \sin \psi, \quad \tau_{r}^{*}=\rho g D \cos \psi \tan \phi^{*}
$$

The shear strength (which depends of the nominal load) in mode II of the simulated weak layer is shown in Extended Data Fig. 4 as a function of slope angle. The loss of contact during weak layer collapse results in an effective weak layer friction angle $\phi^{*} \leq \phi$. The value of $\phi^{*}$ is obtained by fitting Eq. 17 to simulation results for different collapse heights $h$ which leads to the relation between $\phi^{*}$ and $h$ shown in Fig. 2 , 


\section{References}

1. David M McClung. Shear fracture precipitated by strain softening as a mechanism of dry slab avalanche release. Journal of Geophysical Research: Solid Earth, 84(B7):3519-3526, 1979.

2. Jürg Schweizer, Benjamin Reuter, Alec Van Herwijnen, and Johan Gaume. Avalanche release 101. In Proceedings ISSW, pages 1-11, 2016.

3. Johan Gaume, Alec van Herwijnen, Guillaume Chambon, Nander Wever, and Jürg Schweizer. Snow fracture in relation to slab avalanche release: critical state for the onset of crack propagation. The Cryosphere, 11(1):217-228, 2017.

4. A Van Herwijnen and J Heierli. Measurement of crack-face friction in collapsed weak snow layers. Geophysical Research Letters, 36(23), 2009.

5. B Crane Johnson, J Bruce Jamieson, and Robert R Stewart. Seismic measurement of fracture speed in a weak snowpack layer. Cold Regions Science and Technology, 40(1-2):41-45, 2004.

6. Dave Gauthier and Bruce Jamieson. Evaluation of a prototype field test for fracture and failure propagation propensity in weak snowpack layers. Cold Regions Science and Technology, 51(2-3):87-97, 2008.

7. A Van Herwijnen and B Jamieson. Snowpack properties associated with fracture initiation and propagation resulting in skier-triggered dry snow slab avalanches. Cold Regions Science and Technology, 50(1-3):13-22, 2007.

8. J Heierli, P Gumbsch, and M Zaiser. Anticrack nucleation as triggering mechanism for snow slab avalanches. Science, 321(5886):240-243, 2008. 
9. Raymond C Fletcher and David D Pollard. Anticrack model for pressure solution surfaces. Geology, 9(9):419-424, 1981.

10. Johan Gaume, T Gast, J Teran, A van Herwijnen, and C Jiang. Dynamic anticrack propagation in snow. Nature communications, 9(1):1-10, 2018.

11. David Hamre, Ron Simenhois, and K Birkeland. Fracture speed of triggered avalanches. In Proceedings ISSW, pages 174-178, 2014.

12. A van Herwijnen, $\mathbf{J}$ Schweizer, and $\mathbf{J}$ Heierli. Measurement of the deformation field associated with fracture propagation in weak snowpack layers. J. Geophys. Res., 115(F3), 2010.

13. Kristoffer T Walker and Peter M Shearer. Illuminating the near-sonic rupture velocities of the intracontinental kokoxili mw 7.8 and denali fault mw 7.9 strike-slip earthquakes with global p wave back projection imaging. Journal of Geophysical Research: Solid Earth, 114(B2), 2009.

14. Han Bao, Jean-Paul Ampuero, Lingsen Meng, Eric J Fielding, Cunren Liang, Christopher WD Milliner, Tian Feng, and Hui Huang. Early and persistent supershear rupture of the 2018 magnitude 7.5 palu earthquake. Nature Geoscience, 12(3):200-205, 2019.

15. Huihui Weng and Jean-Paul Ampuero. Continuum of earthquake rupture speeds enabled by oblique slip. Nature Geoscience, 13(12):817-821, 2020.

16. Hadar Shlomai and Jay Fineberg. The structure of slip-pulses and supershear ruptures driving slip in bimaterial friction. Nature Communications, 7(1):1-7, 2016.

17. Oded Ben-David, Gil Cohen, and Jay Fineberg. The dynamics of the onset of frictional slip. Science, 330(6001):211-214, 2010. 
18. Elsa Bayart, Ilya Svetlizky, and Jay Fineberg. Fracture mechanics determine the lengths of interface ruptures that mediate frictional motion. Nature Physics, 12(2):166-170, 2016.

19. AJ Rosakis, O Samudrala, and D Coker. Cracks faster than the shear wave speed. Science, 284(5418):1337-1340, 1999.

20. François X Passelègue, Alexandre Schubnel, Stefan Nielsen, Harsha S Bhat, and Raùl Madariaga. From sub-rayleigh to supershear ruptures during stick-slip experiments on crustal rocks. Science, 340(6137):1208-1211, 2013.

21. David S Kammer, Ilya Svetlizky, Gil Cohen, and Jay Fineberg. The equation of motion for supershear frictional rupture fronts. Science advances, 4(7):eaat5622, 2018.

22. Kaiwen Xia, Ares J Rosakis, and Hiroo Kanamori. Laboratory earthquakes: The subrayleigh-to-supershear rupture transition. Science, 303(5665):1859-1861, 2004.

23. Robert Burridge. Admissible speeds for plane-strain self-similar shear cracks with friction but lacking cohesion. Geophysical Journal International, 35(4):439-455, 1973.

24. DJ Andrews. Rupture velocity of plane strain shear cracks. Journal of Geophysical Research, 81(32):5679-5687, 1976.

25. C Sigrist. Measurements of fracture mechanical properties of snow and application to dry snow slab avalanche release. PhD thesis, ETH Zürich, 2006.

26. Y. Liu and N. Lapusta. Transition of mode ii cracks from sub-rayleigh to intersonic speeds in the presence of favorable heterogeneity. Journal of The Mechanics and Physics of Solids, $56: 25-50,2008$.

27. Eran Sharon, Steven P Gross, and Jay Fineberg. Local crack branching as a mechanism for instability in dynamic fracture. Physical Review Letters, 74(25):5096, 1995. 
28. A.C Palmer and J.R Rice. The growth of slip surfaces in the progressive failure of overconsolidated clay. Proc. R.Soc. London, 332:527-548, 1973.

29. J. Gaume, G. Chambon, N. Eckert, and M. Naaim. Influence of weak-layer heterogeneity on snow slab avalanche release: Application to the evaluation of avalanche release depths. J. Glaciol., 59(215):423-437, 2013.

30. Alec van Herwijnen, Johan Gaume, Edward H Bair, Benjamin Reuter, Karl W Birkeland, and Jürg Schweizer. Estimating the effective elastic modulus and specific fracture energy of snowpack layers from field experiments. Journal of Glaciology, 62(236):997-1007, 2016.

31. Ethan Greene, KW Birkeland, K Elder, G Johnson, C Landry, I McCammon, M Moore, D Sharaf, C Sterbenz, B Tremper, et al. Snow, weather, and avalanches: Observational guidelines for avalanche programs in the united states. American Avalanche Association, Pagosa Springs, Colorado, 150, 2004.

32. Bastian Bergfeld, Alec van Herwijnen, Benjamin Reuter, Grégoire Bobillier, Jürg Dual, and Jürg Schweizer. Dynamic crack propagation in weak snowpack layers: Insights from high-resolution, high-speed photography. The Cryosphere Discussions, pages 1-21, 2021.

33. E Bair, J Gaume, and A van Herwijnen. The role of collapse in avalanche release: review and implications for practitioners and future research. In Proceedings of the International Snow Science Workshop, Breckenridge, CO, USA, 02 October 2016, pages 24-31, 2016.

34. J. Gaume, G. Chambon, N. Eckert, M. Naaim, and J. Schweizer. Influence of weak layer heterogeneity and slab properties on slab tensile failure propensity and avalanche release area. The Cryosphere, 9(2):795-804, 2015.

35. Eric M Dunham. Conditions governing the occurrence of supershear ruptures under slipweakening friction. Journal of Geophysical Research: Solid Earth, 112(B7), 2007. 
36. Bruce D Lucas, Takeo Kanade, et al. An iterative image registration technique with an application to stereo vision. Vancouver, British Columbia, 1981.

37. Hao-Yu Wu, Michael Rubinstein, Eugene Shih, John Guttag, Frédo Durand, and William Freeman. Eulerian video magnification for revealing subtle changes in the world. $A C M$ transactions on graphics (TOG), 31(4):1-8, 2012.

38. Michael Lehning, Ingo Volksch, David Gustafsson, Tuan Anh Nguyen, Manfred Stahli, and Massimiliano Zappa. Alpine3d: a detailed model of mountain surface processes and its application to snow hydrology. Hydrological Processes, 20(10):2111-2128, 2006.

39. Torsten Geldsetzer and JB Jamieson. Estimating dry snow density from grain form and hand hardness. In Proceedings International Snow Science Workshop, Big Sky, Montana, USA, pages 1-6, 2000.

40. J Schweizer and C Camponovo. The temperature dependence of the effective elastic shear modulus of snow. Cold Reg. Sci. Technol., 35:55-64, 2002.

41. H Narita. Mechanical behaviour and structure of snow under unaxial tensile stress. $J$. Glaciol., 26(94):275-282, 1980.

42. M Mellor. A review of basic snow mechanics. IAHS Publication, 114:251-291, 1975.

43. M. Mellor and J.H. Smith. Strength studies on snow. Proc. of the International Symposium on Scientific Aspects of Snow and Ice Avalanches, 1966.

44. Alexey Stomakhin, Craig Schroeder, Lawrence Chai, Joseph Teran, and Andrew Selle. A material point method for snow simulation. ACM Transactions on Graphics (TOG), 32(4):1-10, 2013. 
45. D.M. McClung. Shear fracture precipitated by strain softening as a mechanism of dry slab avalanche release. J. Geophys. Res., 84(B7):3519-3526, 1979.

46. B.M Chiaia, P Cornetti, and B Frigo. Triggering of dry snow slab avalanches: stress versus fracture mechanical approach. Cold Reg. Sci. Technol., 53:170-178, 2008.

47. Johan Gaume, Alec van Herwijnen, Ted Gast, Joseph Teran, and Chenfanfu Jiang. Investigating the release and flow of snow avalanches at the slope-scale using a unified model based on the material point method. Cold Regions Science and Technology, 168:102847, 2019.

\section{Acknowledgments}

Funding: J.G. acknowledges financial support from the Swiss National Science Foundation (SNF) grant PCEFP2_181227. We acknowledge Bastian Bergfeld and Stephanie Mayer for the evaluation of the slab density in the full-scale avalanche based on manual snow profiles and SNOWPACK simulations. We acknowledge Jean-François Molinari for helpful and constructive discussions on the topic of supershear crack propagation.

Author contributions: B.T. performed and analyzed the numerical simulations, developed the analytical model, and made all the figures for the paper under the supervision of J.G. and A.vH. R.S. developed the video analysis method and evaluated the crack propagation speed in the avalanche. G.B. analyzed the PST experiments, and A.vH designed and performed the PST experiments. C.J. developed the MPM model. J.G. conceived the key idea of the study and developed the original anticrack elastoplastic model, which was later modified by B.T. B.T. wrote the paper with J.G., including comments from all coauthors.

Competing interests: The authors declare no competing interests. 
Data and material availability: PST and avalanche data as well as simulation results can be found at XXXX. A description of the MPM model can be found in a previous publication at https://www.nature.com/articles/s41467-018-05181-w. 

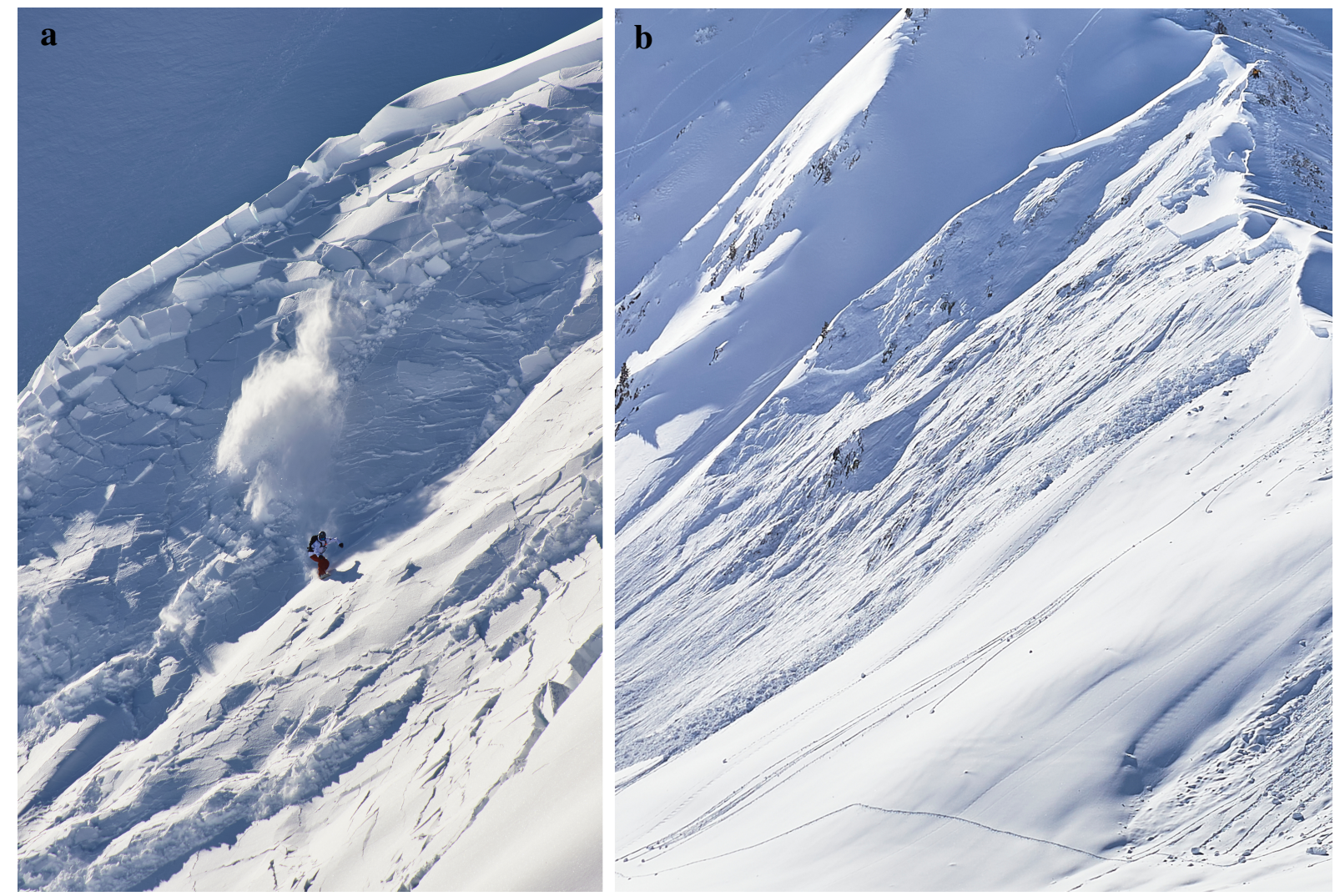

Extended Data Figure 1: a, Snowboarder successfully escaping the release zone after triggering the avalanche. $\mathbf{b}$, Situation of the slope after the avalanche. 

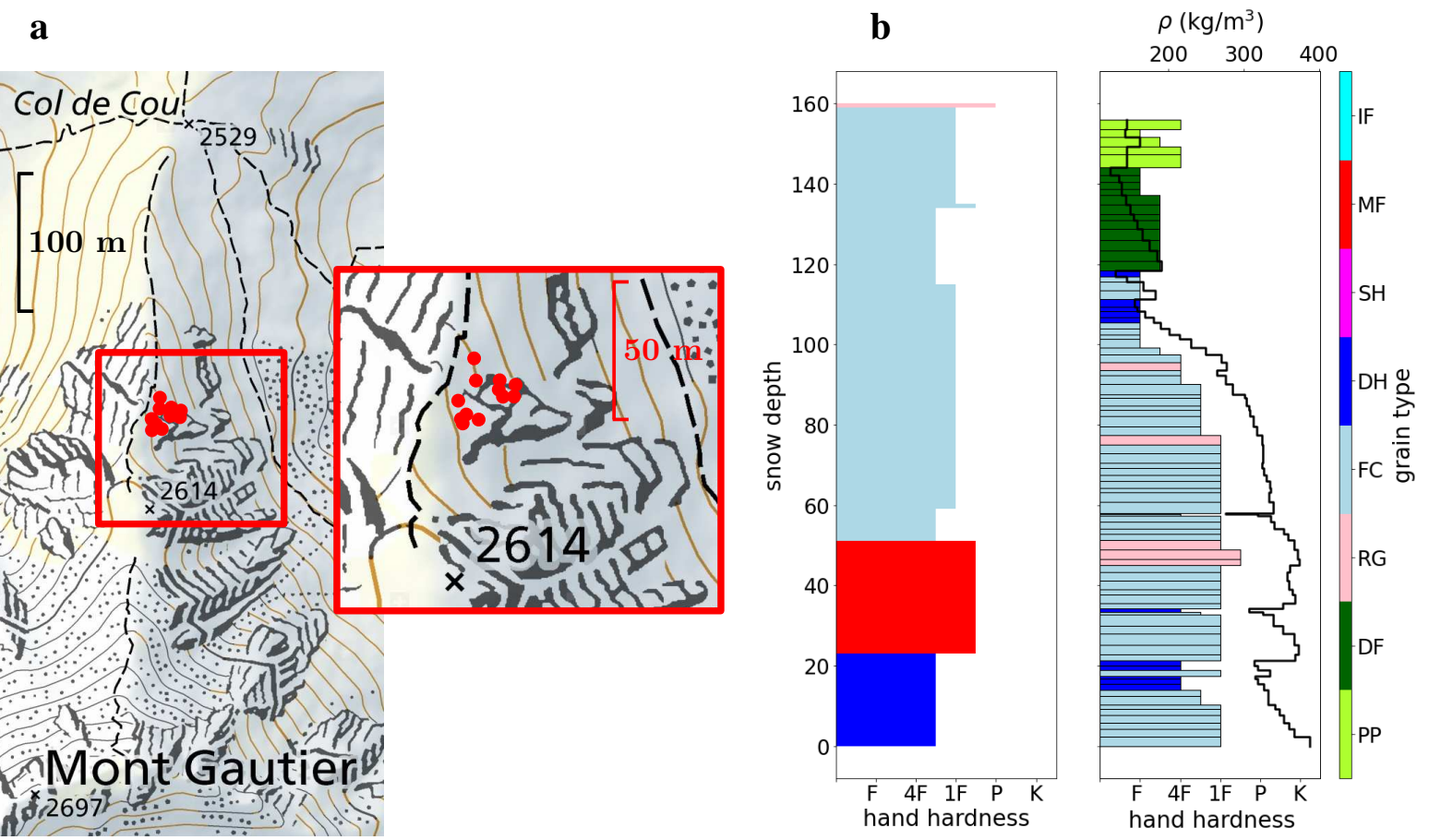

Extended Data Figure 2: a, Location of the avalanche site on the geo.admin.ch portal and position of key-points determined based on the video analysis method. b. Manual snow profile (left) and SNOWPACK simulation (right) showing density and grain size of the different layers. 


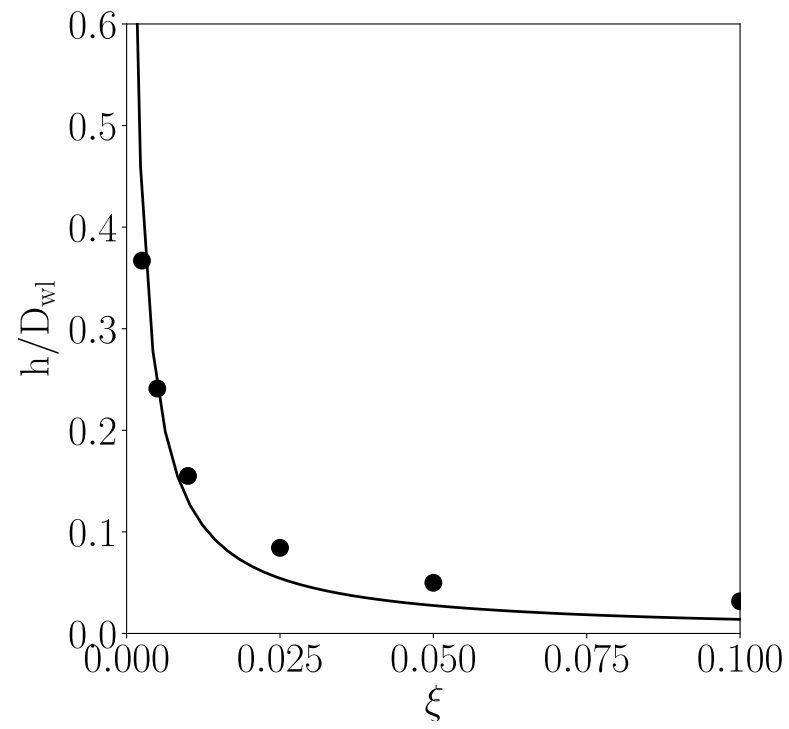

Extended Data Figure 3: Relationship between relative collapse height and hardening coefficient 


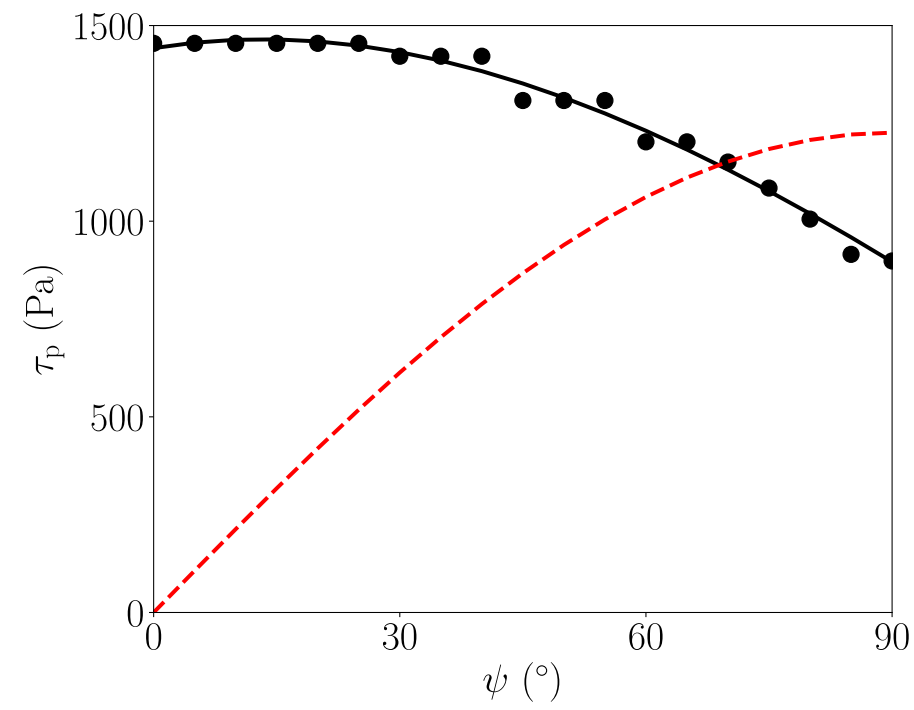

Extended Data Figure 4: Shear strength $\tau_{p}$ of the simulated weak layers as a function of slope angle $\psi$ (black dots), fitted value (black line) and static shear stress $\tau_{g}$ (dashed red line). 


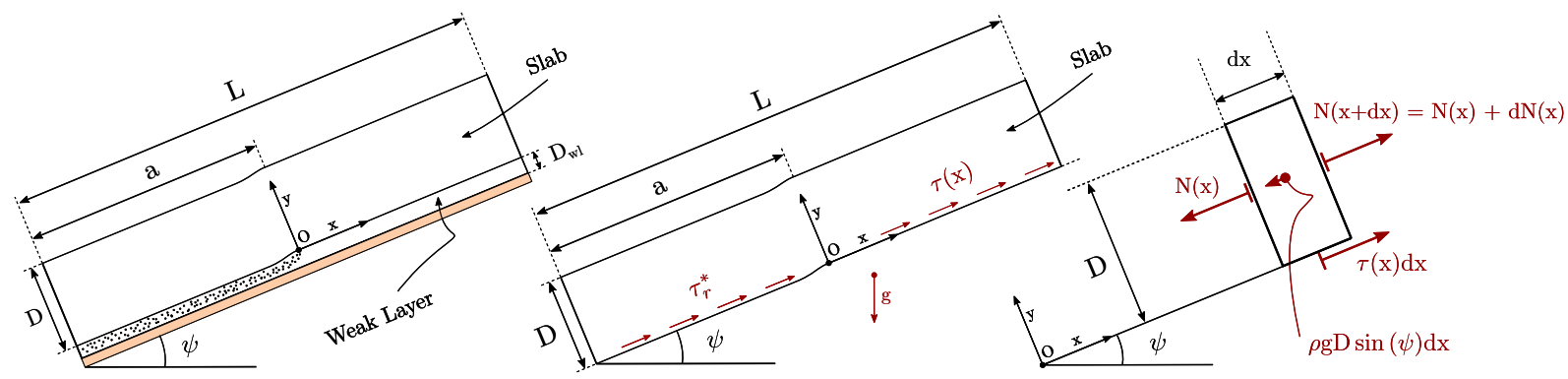

Extended Data Figure 5: Illustration of the system and force balance related to the analytic model for the onset of supershear propagation. 

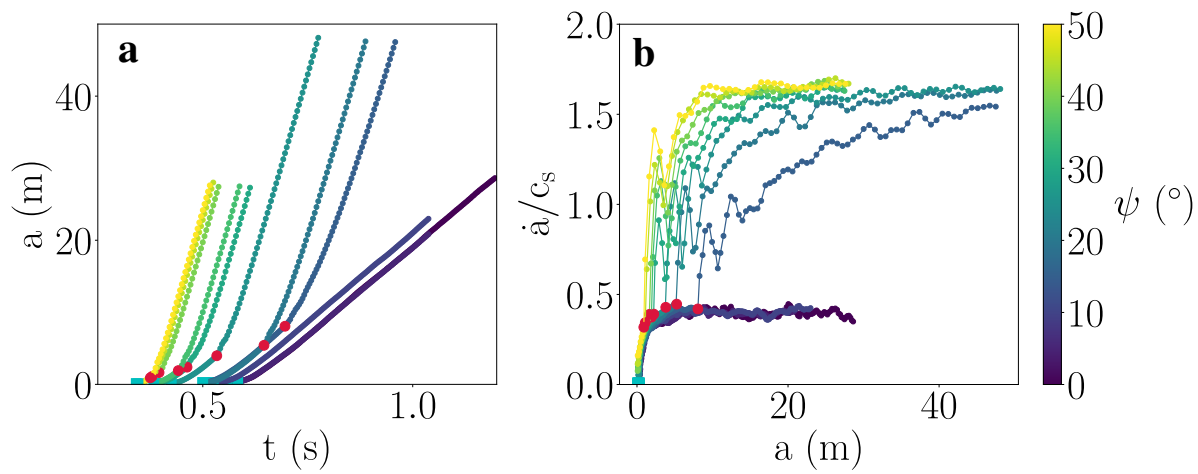

Extended Data Figure 6: Results of PST numerical simulations obtained for slope angles between 0 and $50^{\circ}$.a, temporal evolution of the crack tip location. Blue squares and red circles corresponds respectively to critical and super critical crack lengths. $\mathbf{b}$, normalized crack propagation speed as a function of slope angle. The transition to the supershear regime requires a smaller super critical crack length for larger slope angles. A steeper slopes induces a shorter transient regime. 

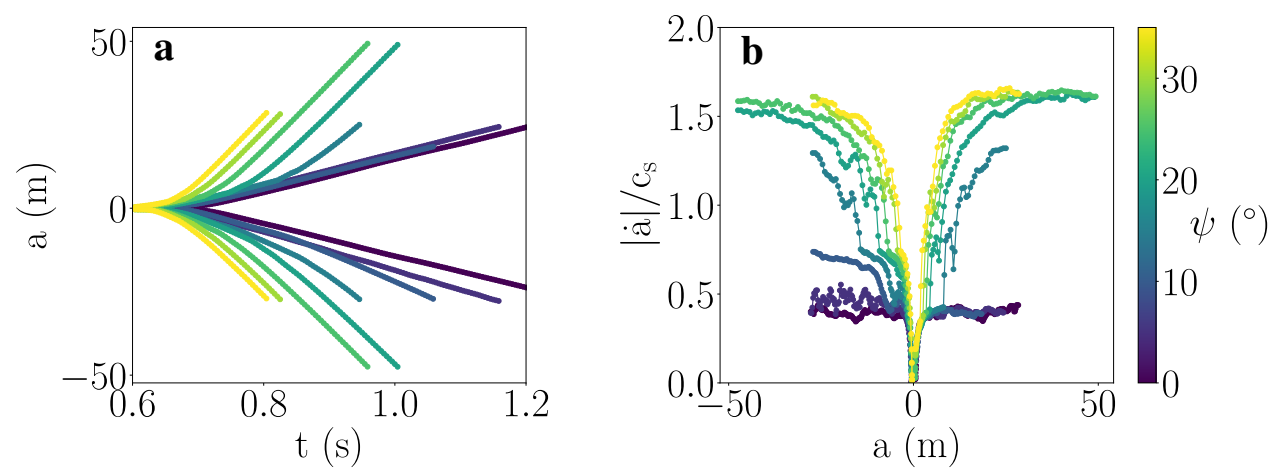

Extended Data Figure 7: Effect of slope angle on the propagation dynamics in Centered Propagation Saw Tests (CPSTs), i.e. with a crack initiated in the middle of the slope within the weak layer. a, temporal evolution of the crack tip location, b, normalized crack propagation speed as a function of the crack length for different values of slope angle. This setup induces propagation in both upslope and downslope directions. While the complex interplay between slab bending induced by weak layer collapse and slab tension (upslope) or slab compression (downslope) breaks the propagation symmetry, the asymptotic speeds are essentially the same in both directions. 

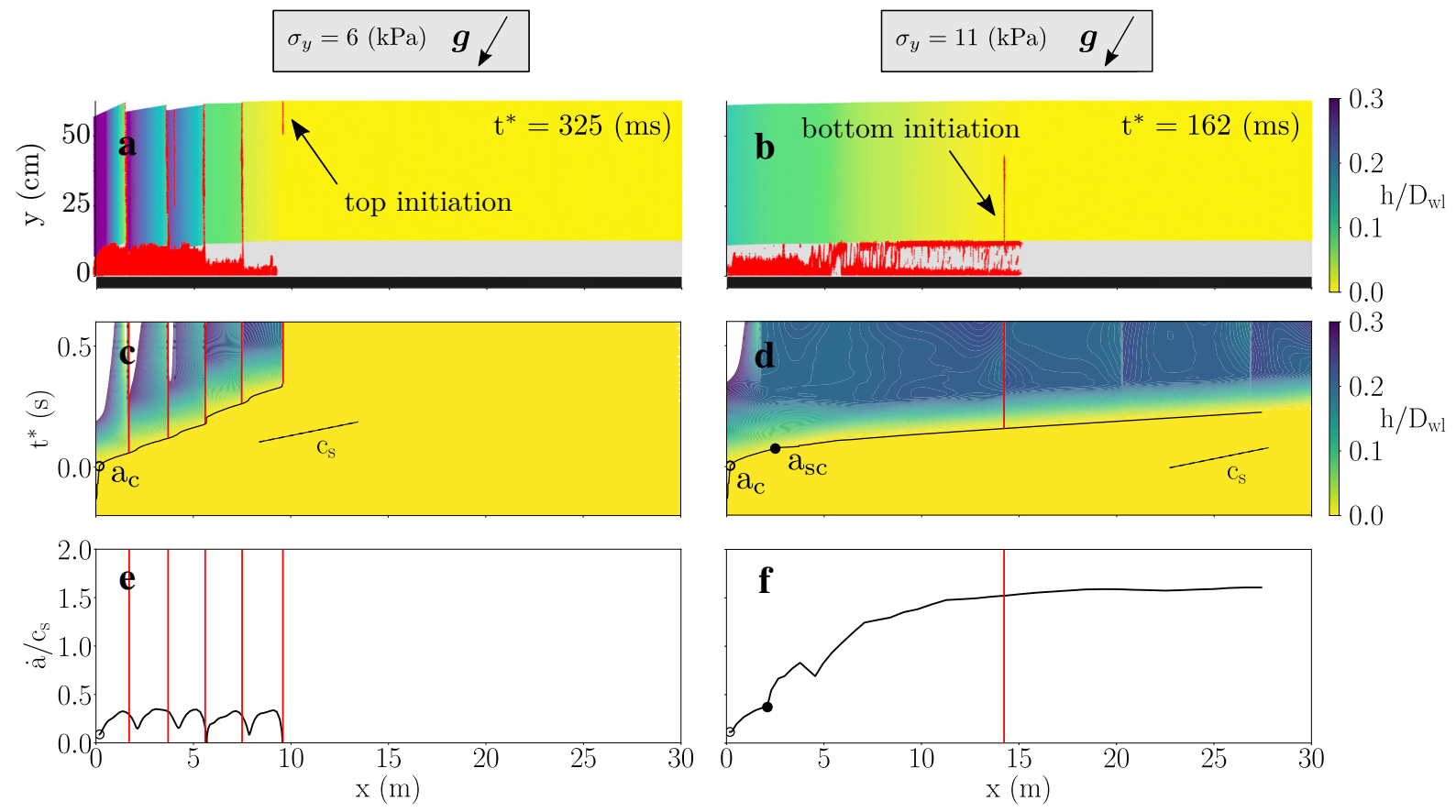

Extended Data Figure 8: Outcome of the simulation shown in Fig. 10, (supershear transition for a $30^{\circ}$ slope) in which we account for slab tensile failure for a relatively soft ( $\sigma_{t}=6 \mathrm{kPa}$, left) and hard ( $\sigma_{t}=11 \mathrm{kPa}$, right) slab. a, b: PST colored by normalised vertical displacement (slab) and weak layer fracture (in red) at two different times. c, d: spatio-temporal evolution of $h / D_{\mathrm{wl}}$ and crack tip location. Black solid lines: crack tip position; empty circles: critical crack length $a_{c}$; filled circles: super critical crack length $a_{s c}$. Red lines: slab fracture. e, f: normalized propagation speed. This figure shows that the tensile failure of soft slab can prevent the supershear transition (panels a and $\mathbf{c}$ ). In this case, the volumetric collapse of the weak layer drives anticrack propagation because the slab cannot accumulate the necessary amount of strain energy required for supershear fracture. This leads to the so-called 'en-echelon' type of fracture ${ }^{33447}$. The crack propagation speed in this case is varying within a sub-Rayleigh range (panel e). The propagation speed decreases locally after the onset of slab tensile failure. Eventually, crack propagation is stopped after an ultimate slab fracture. In contrast, for harder slabs, slab tensile failure may occur but does not perturb crack propagation dynamics and supershear fracture (Panels $\mathbf{b}$ and $\mathbf{d}$ ). In that case, the crack propagation speed is almost identical to that obtain for an elastic slab (Fig. [1b). 


\section{Captions for Movies S1 to S5}

\section{Supplementary Movie S1 - Effect of slope angle on crack propagation dynamics}

Crack tip morphology and dynamics for two MPM simulations (Top: $\psi=0^{\circ}$; Bottom: $\psi=$ $30^{\circ}$ ). The colormap of the slab represents the relative collapse height. Weak layer fracture is indicated in red.

\section{Supplementary Movie S2 - Video of the accidentally triggered snow slab avalanche}

Complete video of the release and flow of the snow slab avalanche analyzed in this study.

\section{Supplementary Movie S3 - Analysis of the avalanche video}

Frame by frame analysis of the change in pixel intensity from the avalanche video.

\section{Supplementary Movie S4 - Effect of slab fractures on crack propagation dynamics}

Crack tip morphology and dynamics for two MPM simulations (Top: $\sigma_{t}=6 \mathrm{kPa}$; Bottom: $\sigma_{t}=$ $11 \mathrm{kPa}$ ). The colormap of the slab represents the relative collapse height. Weak layer and slab fracture are indicated in red.

\section{Supplementary Movie S5 - Centered Propagation Saw Test simulations}

Crack tip morphology and dynamics for two MPM simulations of a Centered Propagation Saw Test (Top: $\psi=0^{\circ}$; Bottom: $\psi=30^{\circ}$ ). The colormap of the slab represents the relative collapse height. Weak layer fracture is indicated in red. 


\section{Supplementary Files}

This is a list of supplementary files associated with this preprint. Click to download.

- SupplementaryMovie1.mp4

- Supplementarymovie2cut.mp4

- SupplementaryMovie3.mp4

- SupplementaryMovie4.mp4

- SupplementaryMovie5.mp4 